\title{
Regulating the Antibiotic drug release from $\beta$-tricalcium phosphate ceramics by atmospheric plasma surface engineering
}

\author{
C. Canal ${ }^{a, b}+$, M. Modic ${ }^{c}$, U. Cvelbar ${ }^{c}$ and M.-P. Ginebra ${ }^{a, b, d}$
}

\begin{abstract}
Calcium phosphate (CaP) ceramics are of interest in bone substitution due to their good biocompatibility and bioresorbability. Currently certain CaPs on the market are loaded with antibiotics in order to prevent infections but further control is needed over the antibiotic release patterns. Cold plasmas have emerged as useful means of modifying the interactions with drugs through surface modification of ie. polymer materials. In this work we explore the possibility of using atmospheric pressure plasma as a tool for the surface modification of these CaP materials with newly populated bonds and charges, with views on enabling higher loading and controlled drug release. Herein the surface modification of tricalcium phosphate ceramics is investigated using atmospheric pressure Helium plasma jet as a tool for tuning the controlled release of the antibiotic Doxycycline hyclate, employed as a drug model. The surface chemistry is tailored mainly by plasma jet surface interaction with increasing the $\mathrm{O} / \mathrm{C}$ ratio without changes in topography as well as by build-up of surface charge. With this surface tailoring it is demonstrated that atmospheric plasma jet is a new promising tool that leads to design of control for drug release from bioceramic matrices.
\end{abstract}

\section{Introduction}

In the field of bone regeneration, there are several classes of synthetic bone grafting biomaterials wherein calcium phosphate bioceramics play a significant role due to their excellent biocompatibility and resorbability. ${ }^{[1]}$ Bone grafting procedures are often performed following bone infections such as osteomyelitis or periodontitis which result in bone loss. The local adminstration of antibiotics in combination with a calcium phosphate delivery matrix with osteoconductive or osteoinductive properties may allow combating against the infection while simultaneously promoting bone regeneration. In this approach, it is essential not only to ensure drug access to the specific bone site, but also to maintain the drug concentration released within the therapeutical range for long periods of time. ${ }^{[2]}$ Therefore, the drug elution kinetics must be carefully controlled.

Calcium phosphate ceramics, which are prepared at high temperatures, must be loaded with drugs commonly by impregnation techniques using highly concentrated solutions. In those materials, the kinetics of release is mainly ruled by the diffusion process, through their structure. However it is not easy to modify such drug release patterns by tailoring the textural properties (surface area, pore size, pore volume, pore shapes, etc.) of ceramic materials treated at high temperatures with wet chemical treatments. $^{[3]}$ Whereas herein we investigate a new strategy to modify surfaces and change the drug release patterns of Calcium Phosphate ceramics with gas discharges. Within the proposed novel approach we evaluate the surface modification of calcium phosphate ceramics with an atmospheric pressure plasma jet (APPJ) for the design of controlled release matrices which would have slower elution patterns. APPJ have small dimensions and could easily be implemented in a surgical room. Thus, this treatment could be compatible with the prophylactic strategy often used by surgeons to prevent post-surgical infections which consists in soaking the synthetic bone graft in an antibiotic solution right before implantation.

Generally, atmospheric pressure plasmas are an emerging technology in the medical field with varied applications ranging from sterilization of medical equipment, surface modification of biomaterials, to treatment of wounds and tissues. ${ }^{[4]}$ The biggest advantage of this technology is treatment of surfaces in ambient air at open atmosphere, which requires neither expensive vacuum equipment and systems nor complicated procedures.

Low pressure plasmas have shown that it is possible to introduce new reactive groups on biomaterials through functionalization or plasma polymerization to obtain the modulation of release of active principles from either polymeric matrices or from model surfaces. ${ }^{[5]}$ To the best of our knowledge, no work has been performed on the application of atmospheric pressure plasma jets to surface modifications of drug release properties from CaP materials.

For this reason, we investigated and evaluated the physic-chemical effects of an APPJ using Helium as carrier gas on the surface properties of microporous $\beta$-Tricalcium Phosphate ( $\beta$-TCP) ceramics. The plasma treated microporous $\beta$-TCP ceramics have been loaded with Doxycycline hyclate (Doxy), an antibiotic with strong activity against periodontal pathogens and with broadspectrum antibiotic effects. ${ }^{[6]}$ The results of the surface modification were also assessed on the in vitro release of Doxy from the ceramics. 


\section{Results}

The atmospheric plasma employed in this work projects a very thin jet (of 1-2 mm diameter of visible light emission) towards the sample (Figures 1a and 1b). To investigate whether the parameters selected (distance to the sample, gas flow and power) would be sufficient to uniformly treat the $15 \mathrm{~mm}$ diameter surface of the $\beta$-TCP discs, static contact angle was envisaged as an adequate technique as it allows measuring different points of the same sample. However, the microporous structure of the $\beta$ TCP ceramics, as well as its inherent hydrophilic properties did not allow measurement of the contact angle due to immediate water absorption by the material. Nevertheless, given the small dimensions of the plasma jet, it was relevant to investigate whether the treatment performed was uniform on the whole treated surface. Therefore, to have an indication of the surface uniformity of the atmospheric plasma treatment, PLLA films were used as model surface and the contact angle was measured at different distances from the center of the sample where the jet was directly applied. As shown in Figure 1c, the atmospheric plasma jet treatment improved wettability by decreasing contact angle more than 200 in the center of the sample where incidence of the jet was direct. Contact angle progressively increased with the distance to the center of the sample where the jet falls upon until $5 \mathrm{~cm}$ of distance where the plasma species are no longer created in abundant quantities.
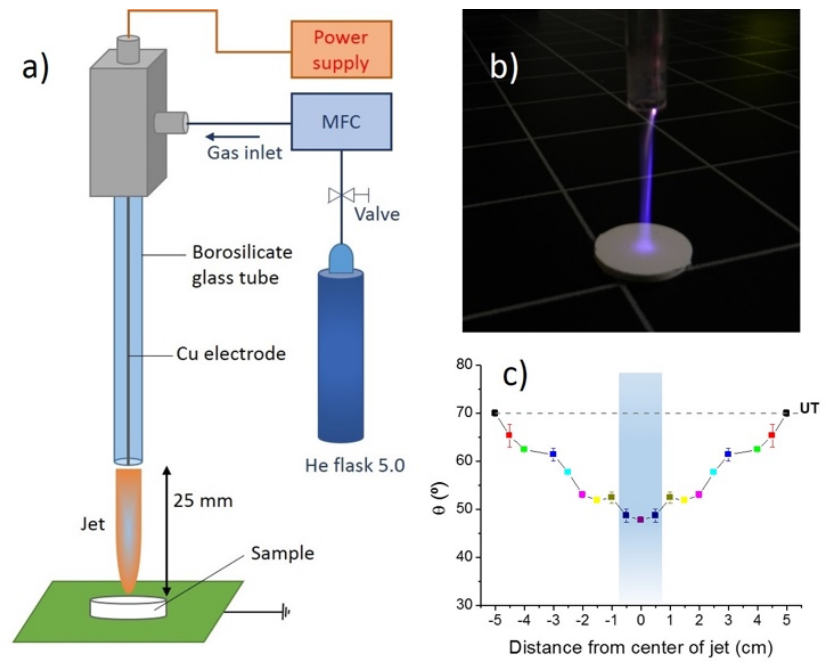

Figure 1. APPJ and uniformity of treatment. Schematic of the experimental set up with APPJ a), photograph of the impinging jet on the $\beta$-TCP discs b), and evaluation of static contact angle on PLLA films as a function of the distance to the plasma jet (He, 20 slm, $10 \min ) \mathrm{c}$ ).

Table 1. Elemental composition and atomic ratio of $\beta$-TCP discs untreated and treated with He APPJ for different times.

\begin{tabular}{|c|c|c|c|c|c|c|}
\hline & \multicolumn{4}{|c|}{ Elemental composition (\%) } & \multicolumn{2}{|c|}{ Atomic ratio } \\
\hline & C & 0 & $\mathrm{Ca}$ & $\mathbf{P}$ & O/C & $\mathrm{Ca} / \mathrm{P}$ \\
\hline UT & 37.40 & 40.60 & 12.90 & 9.10 & 1.09 & 1.42 \\
\hline $1 \mathrm{~min}$ & 28.41 & 45.66 & 14.03 & 10.21 & 1.61 & 1.37 \\
\hline $5 \mathrm{~min}$ & 22.97 & 48.58 & 14.27 & 9.68 & 2.11 & 1.47 \\
\hline $10 \mathrm{~min}$ & 19.12 & 49.74 & 14.90 & 10.63 & 2.60 & 1.40 \\
\hline $20 \mathrm{~min}$ & 12.88 & 51.11 & 13.87 & 9.65 & 3.97 & 1.44 \\
\hline
\end{tabular}

\section{Effects of plasma jet on $\beta$-TCP}

In the first place, the effects of plasma on the surface topography of $\beta$-TCP ceramics were evaluated by Scanning Electron Microscopy (Figure 2). According to the images, the samples are highly porous, and present the typical structure of sintered ceramics. In some of the images some small particles were observed which was attributed to incomplete sintering of the samples. No etching or any other topographical effects were observed due to the plasma treatment.

Given the relevance of the chemical composition of the samples with regard to latter drug loading, the material was analyzed by IR-ATR and X-ray diffraction (Figure 3). On the one hand, IR-ATR of powders obtained from the surface of the disks allowed determining the presence of the phosphate bands characteristic of $\beta$-TCP, as all the bands obtained corresponded to phosphate 
vibrations, where the wide bands correspond to $v_{3}$ stretching $\left(969-1155 \mathrm{~cm}^{-1}\right)$ and $v_{4}$ bending $\left(540-640 \mathrm{~cm}^{-1}\right){ }^{[8]}$ On the other hand, all XRD peaks did correspond to highly crystalline $\beta$-TCP.

The surface chemistry of the ceramics was studied by X-ray Photoelectron Spectroscopy (Table 1). The presence of $C$ on the surface of the sample can be associated to contamination by adsorbed hydrocarbons. The treatment with the atmospheric plasma needle using $\mathrm{He}$ led to a progressive increase in oxygen contents with longer treatment times. The $\mathrm{Ca} / \mathrm{P}$ ratio was slightly lower than the theoretical one for $\beta$-TCP $\left(\mathrm{Ca}_{3}\left(\mathrm{PO}_{4}\right)_{2}, \mathrm{Ca} / \mathrm{P}\right.$ ratio 1.5), and remained unaltered after the APPJ plasma treatment ${ }^{[9]}$.

a) UT
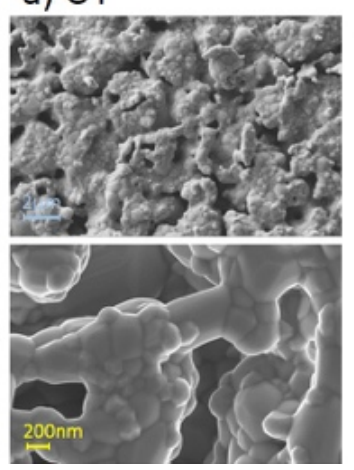

b) $5 \mathrm{~min}$

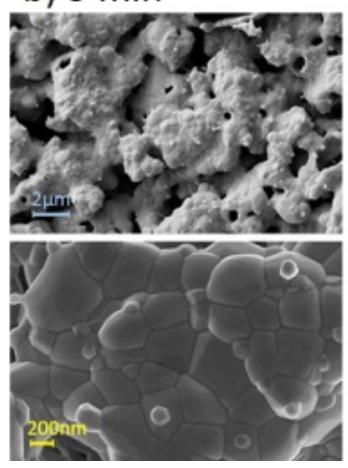

c) $10 \mathrm{~min}$
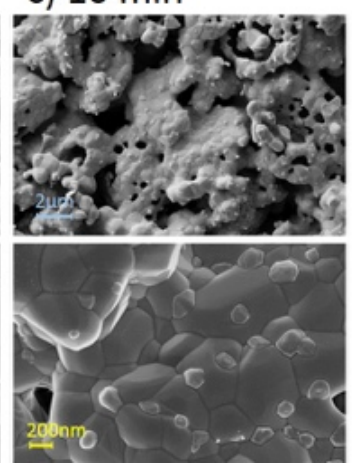

d) $20 \mathrm{~min}$

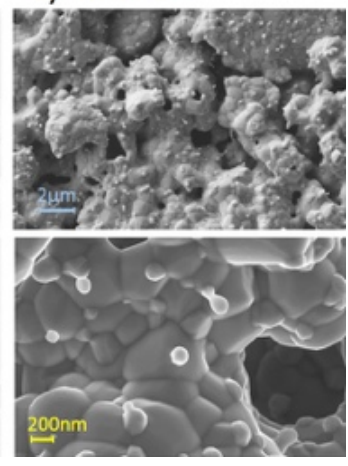

Figure 2. Scanning Electron Micrographs of $\beta$-TCP discs untreated or treated with atmospheric plasma jet for different times at two different magnifications.
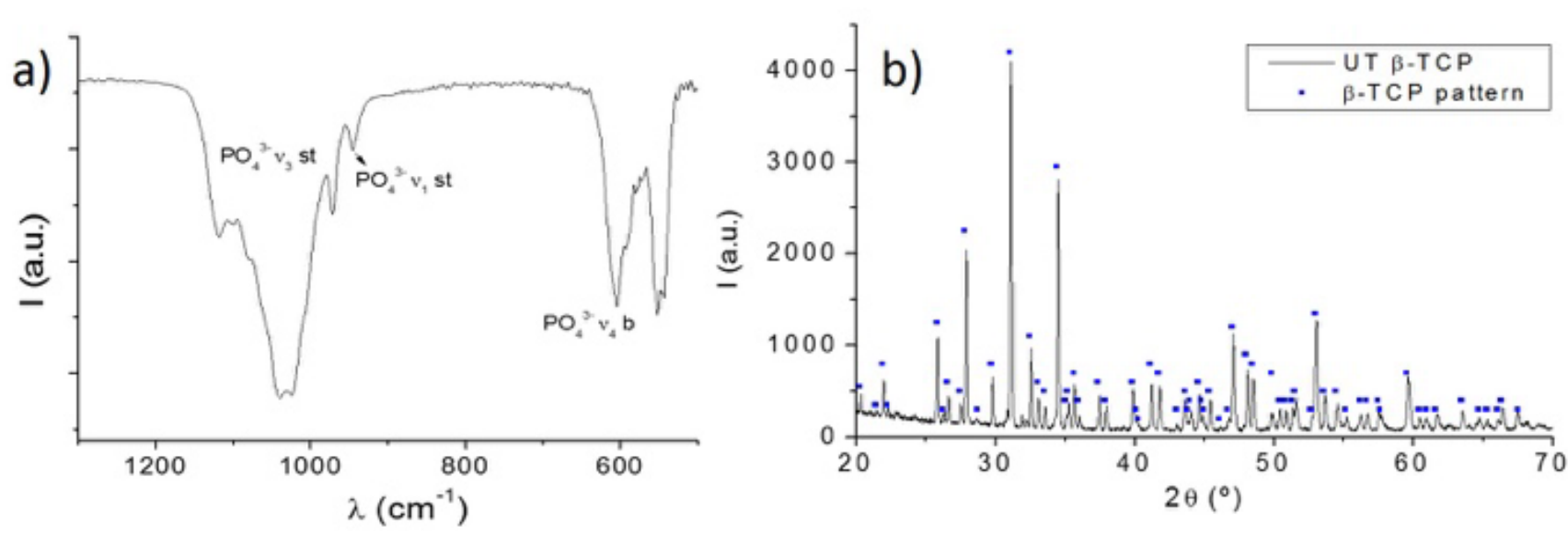

Figure 3. IR-ATR spectra of powders obtained from the surface of untreated $\beta$-TCP (a) and X-ray diffractogram of untreated $\beta$-TCP disks.

Figure 4 presents the deconvolution of the high resolution C1s and O1s XPS spectra of the ceramic surface before and APPJ treatment (20 minutes). The atomic percentages of the carbon and oxygen species are summarized in Table $\mathbf{2}$. Initially, the C1s present on the surface can be ascribed to the presence of adventitious carbon contamination. The O1s peak decomposition suggests that the contaminant possesses hydroxyl groups. With the APPJ treatment the hydrocarbons are progressively eliminated, and at long plasma treatment times there is some extent of functionalization of the hydrocarbons with oxidized moieties such as alcohols, ketones, and carboxyl groups.

Positive charging was measured on the surface of $\beta$-TCP immediately after APPJ treatment (Figure 5). Interestingly, short APPJ treatment times led to charge build up, which seemed to progressively translate inwards the porous structure of the material with increasing treatment times until full surface charge saturation was achieved. This was also observed in ageing graphs (Figures $5 b$ and $5 c$ ) where the $\beta$-TCP ceramics treated for short time showed a decrease of the surface charge with time after treatment, while the samples treated for longer times (e.g. $10 \mathrm{~min}$ ) tended to maintain surface charges for longer times. 

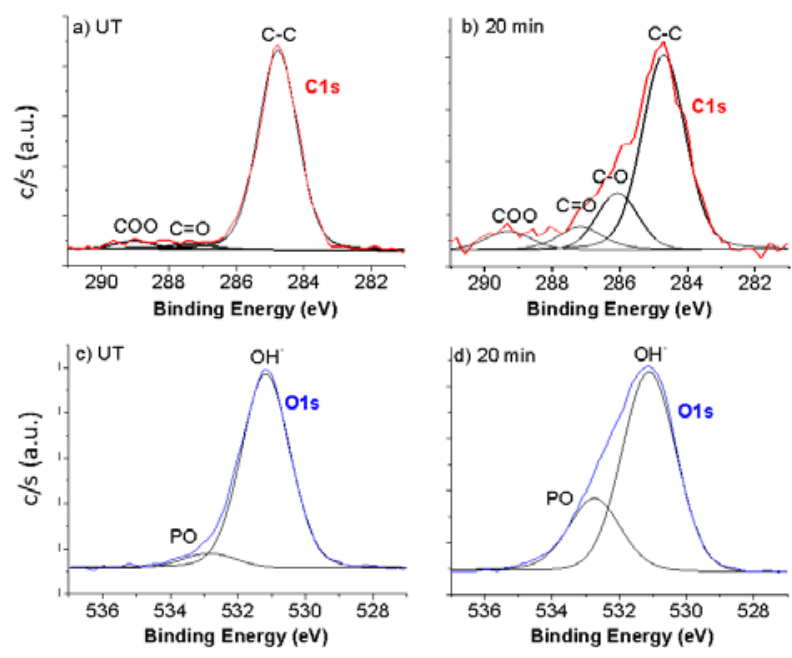

Figure 4. Example of the deconvolution of C1s peak of $\beta$-TCP samples (a) untreated and (b) APPJ treated for 20 minutes and of the deconvolution of O1s peak of $\beta$-TCP samples (c) untreated and (d) APPJ treated for 20 minutes.

Table 2. Decomposition of C1s and O1s peaks of $\beta$-TCP discs untreated and treated with He APPJ for different times.

\begin{tabular}{|c|c|c|c|c|c|c|}
\hline & \multicolumn{6}{|c|}{ Relative chemical composition (\%) } \\
\hline & \multicolumn{4}{|c|}{ C1s } & \multicolumn{2}{|c|}{ 01s } \\
\hline & $284.76 \mathrm{eV}$ & $286.09 \mathrm{eV}$ & $287.09 \mathrm{eV}$ & $288.79 \mathrm{eV}$ & $531.20 \mathrm{eV}$ & $532.90 \mathrm{eV}$ \\
\hline & C-C & C-O & $C=0$ & COO & $\mathrm{OH}^{-}$ & P-O-P \\
\hline UT & 94.25 & 0 & 2.45 & 3.30 & 92.82 & 7.17 \\
\hline $1 \mathrm{~min}$ & 81.89 & 0 & 6.19 & 11.92 & 86.96 & 13.04 \\
\hline $5 \mathrm{~min}$ & 90.29 & 0 & 5.42 & 4.29 & 83.44 & 16.56 \\
\hline $10 \mathrm{~min}$ & 63.43 & 16.86 & 9.13 & 10.59 & 76.63 & 23.37 \\
\hline $20 \mathrm{~min}$ & 66.37 & 17.45 & 9.49 & 6.68 & 72.68 & 27.32 \\
\hline
\end{tabular}

\subsection{Drug loading}

The hydrophilic nature of the $\beta$-TCP ceramics used here led to immediate water absorption. However, dropwise addition of a predetermined volume of aqueous Doxy solution on the samples led to immediate precipitation of the drug for the two concentrations assayed. Figure 6 shows the presence of Doxy crystals completely masking the structure of $\beta$-TCP and blocking the pores of the material, even after APPJ treatment. This impaired the absorption of the drug solution through the pores, so samples were allowed to dry until constant weight before the release experiments. 

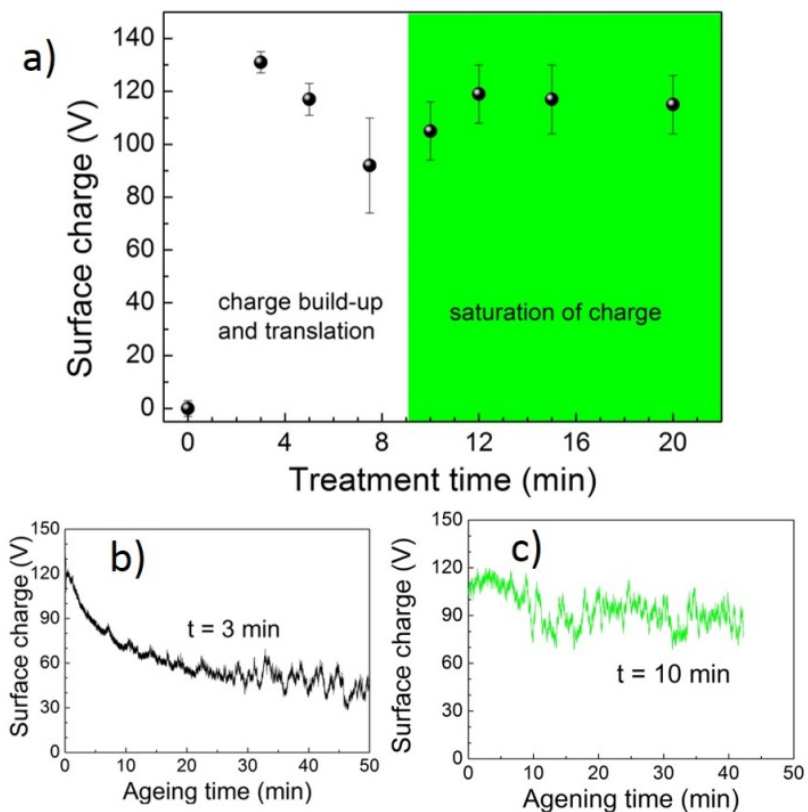

Figure 5. Surface charge of $\beta$-TCP at different treatment times with APPJ (a) and characteristic evolution of surface charge with time after APPJ treatment or so-called surface charge aging in $\beta$-TCP treated for $3 \mathrm{~min}$ (b) or $10 \mathrm{~min}$ (c).
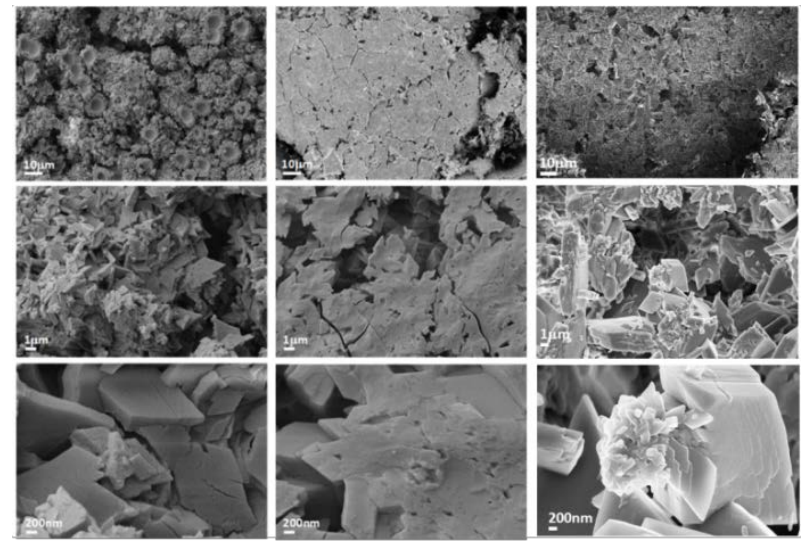

Figure 6. Surface of untreated $\beta$-TCP loaded with two different Doxy amounts ( $3.45 \mathrm{mg}-$ left and $10 \mathrm{mg}-$ center) and APPJ treated and loaded with $10 \mathrm{mg}$ of Doxy at increasing magnifications (from top to bottom).

\subsection{Drug release}

In Figure 7, the cumulative drug percentage released from APPJ treated $\beta$-TCP loaded with two different Doxy concentrations along $24 \mathrm{~h}$ is presented. It can be observed that at both representative concentrations, the untreated samples were those displaying faster initial drug release, which led to higher antibiotic release percentages after $24 \mathrm{~h}$. Whereas interestingly, all plasma treated samples displayed lower release percentages in the initial stages, this effect being typically more pronounced for samples treated for shorter times, regardless of the initial loading concentration tested.
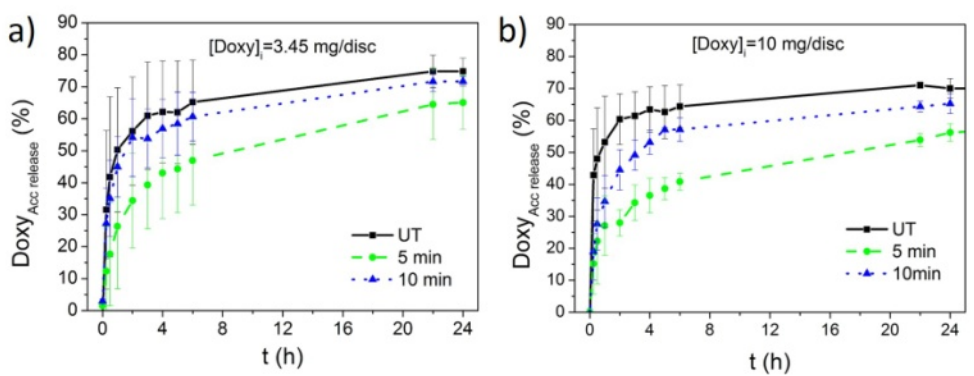

Figure 7. Accumulated Doxycycline release percentage of untreated and APPJ - treated $\beta$-TCP with two different drug loadings (a) 3.45 $\mathrm{mg} /$ disc and (b) $10 \mathrm{mg} /$ disc. 


\section{Discussion}

Atmospheric pressure plasmas are increasingly gaining interest for their wide potential in the medical field ${ }^{[4 a]}$, and their use in the design of biomaterials with improved interactions with other molecules such as drugs or the biological environment is one of the most promising approaches. Herein, we designed a new drug delivery system based on the surface modification of $\beta$-TCP ceramics with an atmospheric pressure plasma. The atmospheric plasma was generated in an APPJ.

which employed He as discharge gas. The He jet features many electrons, He metastables and ions, which are expected to activate the surface of the material directly in the streamer - surface coupling mode, which can create open bonds as well as vacancies in crystal structures. ${ }^{[10]}$ However, it has to be taken into account that apart from the direct physical activation mechanism, some degree of chemical functionalisation might be taking place directly on the sample surface during plasma treatment as well. In fact, in the early afterglow of the plasma, a certain percentage of ambient air is mixing with the He jet and is generating radicals from air. This was already demonstrated in previous works ${ }^{[11]}$, where optical emission spectroscopy was performed and excited He emission lines were observed along with other emission lines corresponding to neutral $\mathrm{O}$ atom, $\mathrm{H} \alpha$, $\mathrm{OH}\left(A^{2} \Sigma-X^{2} \Pi\right), N_{2}{ }^{+}$first negative $\left(C^{3} \Pi_{u}-B^{2} \Sigma_{u}^{+}\right)$and $N_{2}\left(B^{3} \Pi_{u}-B^{3} \Sigma_{u}^{+}\right)$second positive molecular band. Additionally, because of the diffusion of ambient air into the plasma and consequent collisions, the emission intensities of the He lines decrease as the plasma plume propagates into the surrounding air and the intensity of the other species increases.

It has been shown using a model PLLA film that the treatments performed modify the whole diameter of the samples uniformly (Figure 1c). The contact angle measured tended to decrease with the distance from the jet, indicating that the active species react directly where the flow of the plasma is directed, and their density progressively decreases with distance. Although it is well known that different kinds of materials display different recombination probabilities towards the neutral radicals in the plasma phase ${ }^{[12]}$, in general metallic materials are more reactive, showing higher recombination probabilities, while polymers and ceramics display lower reactivity, so the wettability results obtained with the PLLA film can be extrapolated to the $\beta$-TCP material employed here. It has been observed that wettability modifications on a flat surface are visible up to a radius of $5 \mathrm{~cm}$ from the center of the jet, so it can be considered that the samples used, with a radius of $0.75 \mathrm{~cm}$ are uniformly modified by the treatment performed here.

The APPJ treatment performed was mild, avoiding any modification of the surface topography of the ceramics (Figure 2), or its $\mathrm{Ca} / \mathrm{P}$ ratio that was found slightly lower than expected. In previous works this slightly lower $\mathrm{Ca} / \mathrm{P}$ ratio detected was attributed to the fact that XPS analysis is restricted just to the outer surface of the material ${ }^{[13]}$. While plasma treatment of polymeric materials often proceeds through a time-dependent surface functionalisation with oxygen-containing groups (ie. carbonyl and carboxyl) ${ }^{[14]}$, here cleaning effects seemed to be predominant (Tables 1 and 2). The O1s XPS high resolution peak decomposition suggested that the contaminant possesses acidic hydroxyl groups ${ }^{[15]}$ which are progressively removed by the etching effect of the APPJ. Recent works with atmospheric pressure plasmas on hydroxyapatite ceramics ${ }^{[16]}$ found a predominant cleaning effect with very hardly any oxygen functionalisation, as in the present study, while on biphasic hydroxyapatite/ $\beta$-TCP ceramics ${ }^{[17]}$ improved wettability was recorded with air atmospheric pressure plasmas with some extent of oxygen functionalisation, although it was not quantified.

The $\beta$-TCP samples used in this study were highly porous (Figure 2 ) with pore sizes in the order of 0.5 - $2 \mu \mathrm{m}$. Untreated $\beta$-TCP displayed very good water wettability. Unexpectedly, when samples contacted the aqueous Doxy solutions used here, no absorption of the solution was observed. This could be attributed to immediate precipitation of Doxy crystals on the surface of the ceramics, which blocked the pores of the material and hampered absorption of the drug solution by the bioceramic (Figure 6). This phenomenon can be ascribed to the interaction of Doxy with Calcium. In fact, Doxycycline is an antibiotic from the tetracycline family. It is known that tetracyclines display strong adsorption affinity for calcium phosphates ${ }^{[18]}$, and in spite of the lower affinity of Doxy with Ca compared to other tetracyclines, it was previously observed that its interaction slightly delayed the hydrolysis reaction in Calcium Phosphate Cements, affecting different properties. ${ }^{[19]}$ Even though two different drug loads were tested, even the lowest led to a surface completely covered with Doxy crystals, so no penetration of the drug inside the material can be assumed.

The results obtained regarding drug release are highly interesting and open a new pathway for the design of slower-releasing ceramic drug delivery systems. The release of Doxy from the materials took place in a two-step mechanism: an initial stage of burst release, which can be associated with the precipitated Doxy not directly bound to the surface. This was followed by a stage of slower release rate, where the stationary stage was not reached (Figure 7). The untreated samples with both starting Doxy concentrations reached around $60 \%$ of release in the first $3 \mathrm{~h}$, while plasma treated samples had much lower release percentages in the same timeframe with values of $35-40 \%$ in the 5 min treated samples. Additionally, it was observed that the second release stage was delayed at least for $1 \mathrm{~h}$, when the $\beta$-TCP samples had been treated with APPJ, although the release remains still too fast for the intended application. 
The charge conferred by the APPJ to the $\beta$-TCP surface may be related, at least in part, with the release results obtained: Liang et al. reported asymmetric charge distribution on the $\beta$-TCP surfaces with negatively charged areas near the $O$ and positively charged ones in the vicinity of $\mathrm{P}$ and $\mathrm{Ca}$ atoms. ${ }^{[20]}$ Following APPJ treatment positive charging was generated on the surface (Figure 5). This is in agreement with computational models ${ }^{[21]}$ that demonstrated that in the interaction of a He APPJ with a dielectric surface, the flux of positive ions $\left(\mathrm{O}_{2}^{+}\right.$in particular) to the surface is greater than that of negative ions $\left(\mathrm{O}_{2}^{-}\right)$. The strong electric field directed toward the dielectric surface causes the positive ions in the vicinity of the surface to migrate to the dielectric surface, where their positive charge is deposited on the surface of the dielectric. It seemed here that the positive charge diffused with time into the porous $\beta$-TCP structure until saturation was reached at treatment times above 10 min (Figure 5).

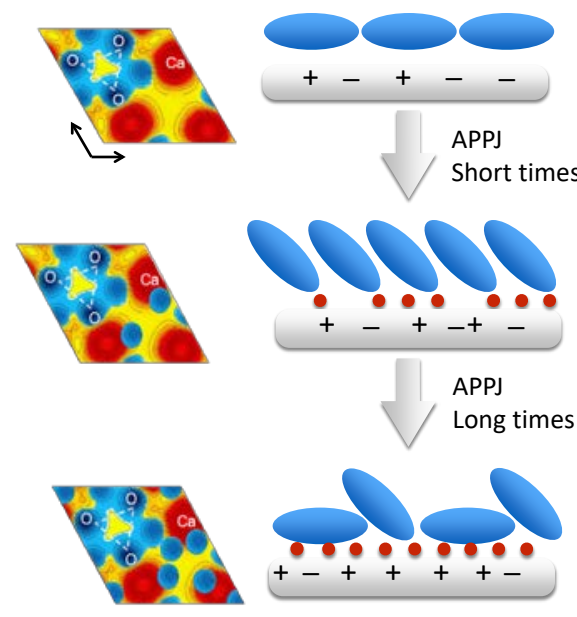

Figure 8. Proposed mechanism of interaction established between Doxycycline and the untreated $\beta$-TCP ceramics or APPJ treated ones at different treatment times. Exposure of O-groups by etching of surface contaminants and some degree of functionalisation progressively takes place with treatment time while the positive surface charge build-up is unstable at short treatment times while it is more permanent at longer times, thus compensating for the higher electronegativity of O-groups and hampering the interactions with Doxy.

It can be speculated that the drug release patterns observed at different APPJ treatment times were a result of the combination of two effects: i) surface cleaning as shown by XPS (Tables 1 and 2) and ii) surface charge build up and stability. Both parameters may influence the spatial placement of the Doxy molecules adsorbed on the surface (Figure 8). As APPJ treatment exposes oxygen from $\beta$-TCP by removal of contaminants and by grafting of oxygen groups on the surface at short treatment times, and the charge created is rapidly generated, interaction of Doxy with the surface is enhanced, possibly through its amine groups, allowing higher packing of molecules on the surface. This improved interaction Doxy $\beta$-TCP may account for the slower drug release recorded. As APPJ treatment proceeds at longer times, the positive charges generated on the surface are more stable and compensate for the higher electronegativity conferred by the O-groups, tending to hamper the interactions with Doxy.

It has been shown in other works that atmospheric pressure plasmas improve wettability of hydroxyapatite ${ }^{[16]}$ and that on calcium phosphate scaffolds they can lead to improved osteoblast attachment and proliferation ${ }^{\text {[17] }}$, so the combination of such features with the slower drug release obtained here can provide the bioceramics with highly attractive features. The strategy introduced in this work opens new perspectives for the control of drug release from ceramic matrices through the use of atmospheric plasmas.

\section{Conclusions}

In the present work the surface of $\beta$-TCP ceramics was conditioned with a He APPJ at different times, which enabled us to optimize and uniformly modify the chemistry of the $\beta$-TCP ceramics without altering their morphological features. Positive surface charge was generated, which was stable for long APPJ treatment times. The antibiotic Doxycycline was incorporated on the APPJ treated side of the materials, and it was demonstrated that the slower release of the antibiotic from the plasma treated ceramics could possibly be ascribed to the combination of higher exposure of $O$ groups from the surface and surface charge build up and stability. With this novel approach it was possible to achieve more than $33 \%$ improvement in retaining drug release for optimized APPJ treated $\beta$-TCP with respect to untreated samples. 


\section{Experimental Section}

\subsection{Material synthesis}

Microporous $\beta$-TCP discs were produced by sintering calcium deficient hydroxyapatite (CDHA) discs ( $\mathrm{Ca} / \mathrm{P}=1.5)$, obtained from a Calcium Phosphate Cement. The cement's solid phase was $\beta$-TCP, obtained by heating in a furnace (Hobersal CNR-58) in air an appropriate mixture of calcium hydrogen phosphate $\left(\mathrm{CaHPO}_{4}\right.$, Sigma Aldrich) and calcium carbonate $\left(\mathrm{CaCO}{ }_{3}\right.$, Sigma-Aldrich) for $15 \mathrm{~h}$ at $1400^{\circ} \mathrm{C}$, followed by quenching in air. $\beta$-TCP powder was milled in an agate ball mill (Pulverisette $6, \mathrm{Fritsch} \mathrm{GmbH}$ ) with 10 balls $(d=30 \mathrm{~mm})$ for $15 \mathrm{~min}$ at $450 \mathrm{rpm}$. The liquid phase employed to prepare the cement consisted of a $2.5 \%$ solution of $\mathrm{Na}_{2} \mathrm{HPO}_{4}$ in MilliQ water. The powder phase was mixed with the liquid phase (L/P ratio of $0.35 \mathrm{ml} / \mathrm{g}$ ) in a mortar for about $1 \mathrm{~min}$ and then transferred into disc moulds of $15 \mathrm{~mm}$ diameter $\times 2 \mathrm{~mm}$ height. Samples were allowed to set in water for 2 days to allow for its transformation into CDHA, before demoulding, drying and sintering. CDHA was transformed into $\beta$-TCP by sintering in air at 1110 ㅇ for $9 \mathrm{~h}$, followed by slow cooling.

\subsection{Experimental methods}

\subsubsection{Atmospheric Plasma Jet treatment}

An APPJ was created using He as plasma carrier gas in an end-filed jet design with electrode. The discharge electrode was a copper wire with a diameter of $0.1 \mathrm{~mm}$ inserted inside of a $1.2 \mathrm{~mm}$ inner diameter quartz capillary tube and was connected to a commercial high voltage power supply from Conrad Electronics (nominally $6 \mathrm{~W}$ power consumption). The discharge was operating with sinusoidal waveform at $23 \mathrm{kHz}$ with $(\mathrm{U}) \sim 2 \mathrm{kV}$ and $(\mathrm{I}) \sim 3 \mathrm{~mA}$. The power input into discharge was about $1 \mathrm{~W}$. Helium of purity 5.0 was leaked into the gas tube and then to ambient atmosphere at 15 slm through a Bronkhorst MassView flow controller. The samples were placed under the jet at equal distances of $25 \mathrm{~mm}$ from the tube opening and electrode end.

\subsubsection{Drug incorporation}

Antibiotic was incorporated to the $\beta$-TCP discs either untreated or plasma treated by addition of $100 \mu$ of aqueous Doxy solutions to the top surface of the samples. The drug was placed on the plasma-treated side of the $\beta$-TCP discs and two different Doxy concentrations were used so as to obtain samples loaded with either $3.45 \mathrm{mg}$ or with $10 \mathrm{mg}$ of Doxy. Samples were allowed to dry at ambient temperature in a desiccator until constant weight.

\subsubsection{Drug release}

The drug release tests were performed according to the USP Pharmacopoeia Dissolution Test in a Paddle Dissolution Tester (Pharma Alliance, USA) with 8 amber vessels of $250 \mathrm{ml}$ with $150 \mathrm{ml}$ Phosphate Buffer Solution (PBS). $\beta$-TCP samples without drug were used as control. Stirring conditions were $150 \mathrm{rpm}$, temperature was set to $37^{\circ} \mathrm{C}$ and $1 \mathrm{~mL}$ aliquots were withdrawn at predetermined times from the receptor compartment for spectroscopy analysis at $\lambda=351 \mathrm{~nm}$ (TECAN, Infinite M200 Pro Microplate Reader). After each sample withdrawal, the same volume of fresh PBS was added to the receptor medium. The concentration of Doxy in the receptor solution was below $20 \%$ of the saturation concentration (sink conditions) during the experiment. ${ }^{[22]}$ Four replicates were studied of each sample $(n=4)$. To precisely determine the Doxycycline concentration at each time point, three corrections were taken into account: (1) A total volume correction due to evaporation during the release experiment; (2) A correction to take into account the drug quantity withdrawn at each sampling; (3) A correction due to the antibiotic degradation in the experiment conditions.

\subsection{Characterization Techniques}

\subsubsection{Wettability Contact Angle Measurements}

Static contact angle was determined on PLA films with an Oca15+ (Dataphysics) using water as wetting liquid and with SCA20 software.

\subsubsection{Scanning Electron Microscopy}

The microstructure of the ceramics was imaged by Field Emission Scanning Electron Microscopy (SEM, JEOL JSM-7001F). Samples were $\mathrm{C}$ sputter coated prior to observation.

\subsubsection{X-Ray Photoelectron Spectroscopy}

The surface chemistry in upmost layers with penetration depth of $20 \mathrm{~nm}$ was investigated by X-ray photoelectron spectroscope (XPS) (TFA XPS Physical Electronics, Chanhassen, Minnesota, USA). The base pressure in the analysis chamber prior the measurements was approximately $6 \times 10^{-8} \mathrm{~Pa}$. We used Al $\mathrm{K}_{\alpha 1,2}$ monochromatic source of X-ray light with energy of $1486.85 \mathrm{eV}$ for sample analyses. The photoelectrons were detected with a hemispherical analyzer positioned at an angle of $45^{\circ}$ with respect to the normal to the sample surface. The samples were analyzed over a $400 \mu \mathrm{m}$ spot area. Each sample was analyzed at two different positions and the average composition was calculated. The relative error for XPS determination was about $0.5 \%$. The XPS spectra were measured for an untreated and helium plasma treated samples. In addition to the wide energy range spectra, high-energy resolution spectra of characteristic peaks of $\mathrm{C}_{1 \mathrm{~s}}, \mathrm{O}_{1 \mathrm{~s}}, \mathrm{~N}_{1 \mathrm{~s}}$ and $\mathrm{Si}_{2 \mathrm{p}}$ elements are recorded through a narrow energy range. Spectra were referenced to the $C_{1 s}$ peak, at $284.80 \mathrm{eV}$. Deconvolution, using Gaussian-Lorentzian fit was performed with MultiPak v7.3.1 software from Physical Electronics, supplied with the spectrometer.

\subsubsection{Infrared-Atenuated Total Reflectance}

Infrared with Attenuated Total Reflectance (IR-ATR) was performed on powder obtained from the surface of $\beta$-TCP discs and placed in a horizontal unit, covered by a germanium crystal. 


\subsubsection{X-Ray Diffraction}

Phase composition of powders obtained from the surface of $\beta$-TCP ceramics either untreated or APPJ treated was assessed by Xray powder diffraction (XRD, PANalytical, X'Pert PRO Alpha-1) by scanning in Bragg-Brentano geometry using CuK $\alpha$ radiation $(\lambda=1.5406 \mathrm{~A})$. The experimental conditions used were: $1.5 \mathrm{~h}$ of scan time with $2 \theta$ scan step of $0.020 \cong$ and scan range between 4

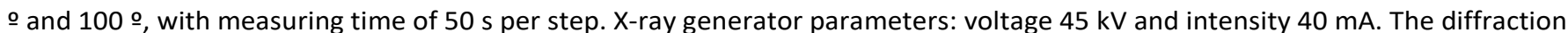
patterns were compared with the Joint Committee on Powder Diffraction Standards for $\beta$-TCP (JCPDS No. 9-169). ${ }^{\text {[23] }}$

5.3.6. Surface charge

For electric field measurements a Trek (model 347, New York, USA) field meter with a proper high voltage probe was used. The field meter is capable of measuring $\pm 3 \mathrm{kV}$ DC voltage with accuracy better than $0.05 \%$ of full scale. The high voltage probe is circular with the diameter of $1.3 \mathrm{~mm}$. A fast digital input card (NI USB 6009) was connected to the field meter via a BNC connector, which enabled to display and record voltage measurements in a time resolution of 50 ms. Surface charge experiments were done in a grounded box. The grounding was important to lower the electrical noise of the surroundings. Prior to conducting measurements, the field meter was zeroed using a grounded steel plate. The distance between the sample and the probe was $1.1 \mathrm{~cm}$.

\section{Acknowledgements}

Authors acknowledge the financial support of the COST Action MP1101 and MINECO for MAT2015-65601-R project, cofunded by the EU through European Regional Development Funds. CC acknowledges MINECO for the Ramon y Cajal fellowship. Support for the research of MPG was received through the ICREA Academia Award for excellence in research, funded by the Generalitat de Catalunya. UC and MM acknowledges support of ARRS agency and NATO SPS 984555 grant.

\section{References}

1 a) S.V. Dorozhkin, Biomatter. 2011, 1, 3; b) J.M. Rueger, Orthopade 1998; 27:72; c) A.S.Greenwald, S.D.Boden, V.M. Goldberg, Y.Khan, C.T. Laurencin, R.N. Rosier, J. Bone Joint Surg. Am. 2001, 83:98. d) C.G. Finkemeier, J. Bone Joint Surg. Am. 2002, 84, 454; e) P.V. Giannoudis, H. Dinopoulos, E. Tsiridis, Injury 2005, 36:20; f) M. Bohner, Mater. Today 2010, 13, 24; g) C. Canal, M.P. Ginebra, J. Mechanical Behav. Biomed. Mater., 2011, 4, 1658; h) M.P. Ginebra, M. Espanol, E.B. Montufar, R.A. Perez, G. Mestres ${ }_{\llcorner}$ Acta biomater. 2010, 6, 2863. i) X. Yu, X. Tang, S.V. Gohil, C.T. Laurencin Adv. Healthcare Mater. 2015, 4, 1268.

2 M.P. Ginebra, C. Canal, M. Espanol, D. Pastorino, E.B. Montufar, Adv. Drug Delivery Rev. 2012, 64, 1090.

3 D. Arcos, M. Vallet-Regí, Acta Materialia, 2013, 61, 890.

4 a) T. von Woedtke, S. Reuter, K. Masur, K.D. Weltmann. Phys. Reports, 2013, 530, 291. b) J. Winter, R. Brandenburg, K.-D. Weltmann, Plasma Sources Sci. Technol. 2015, 24, 064001.

5 a) C. Labay, J.M. Canal, M. Modic, U. Cvelbar, M. Quiles, M. Armengol, M.A. Arbos, F.J. Gil, C. Canal, Biomaterials, 2015, 71, 132; b) C. Labay, J.M. Canal, C. Canal, Plasma Process. Polym., 2012, 9,165; c) S. Bhatt, J. Pulpytel, M. Mirshahi, F. Arefi-Khonsari, Polymer 2013, 54, 4820.

6 K. Parfitt, W. Martindale, in The complete drug reference, Pharmaceutical Press, London, UK 1999.

7 S.V. Dorozhkin, J. Mater. Sci.: Mater. Med., 2007, 42, 1061.

8 a) G. Mestres, C. Le Van, M.P. Ginebra, Acta Biomater. 2012, 8, 1169. b) S.R. Radin, P. Ducheyne, J. Biomed. Mater. Res., 1993, $27,35$.

9 J.C. Elliott, Structure and Chemistry of the Apatites and Other Calcium Ortophosphates. Elsevier, Amsterdam. 1994.

10 U. Cvelbar, Z. Chen, I. Levchenko, R. M. Sheetz, J.B. Jasinski, M. Menon, M. K. Sunkara, K. Ostrikov, Chem. Commun., 2012, 48, 11039.

11 a) R. Zaplotnik et al, EPL 2014, 106,2, 25001; b) R. Zaplotnik et al. Spectrochimica acta Pr. B: Atomic spect. 2015, 103/104, 124.

12 J.P. Sarrette, B. Rouffet, A. Ricard, Plasma Process. Polym., 2006, 3, 120.

13 A. R. Boyd, G. A. Burke, H. Duffy, M. L. Cairns, P. O'Hare, B. J. Meenan, J Mater Sci: Mater Med 2008, $19,485$.

14 a) C.Canal, F.Gaboriau, R.Molina, P.Erra, A.Ricard, Plasma Process. Polym., 2007, 4, 445; b) C. Labay, J.M. Canal, C. Canal, Plasma Process. Polym., 2011, 9, 165.

15 a) R. França, T.D. Samani, G. Bayade, L. Yahia, E. Sacher, J. Colloid and Interface Sci. (2013); b) N. Marin-Pareja, E. Salvagni, J. Guillem-Marti, C. Aparicio, M.P. Ginebra, Colloids Surf B Biointerfaces, 2014, 122, 601.

16 I. Koban, K. Duske, L. Jablonowski, K. Schröder, B. Nebe, R. Sietmann, K.D. Weltmann, N.O. Hübner, A. Kramer, T. Kocher, Plasma Process. Polym., 2011, 8, 975.

17 Y.R. Choi, J.S. Kwon, D.H. Song, E.Ha Choi, Y.K. Lee, K.Na. Kim, K.M. Kim, Thin solid films, 2013, 547, 235.

18 a) A. Ratier, M. Freche, J.L. Lacout, F. Rodriguez, Int. J. Pharm., 2004, 274, 261; b) A. Ratier, I.Gibson, S. Best, M. Freche, J. Lacout, Biomaterials, 2001, 22, 897.

19 C. Canal, D. Pastorino, G. Mestres, P. Schuler, M.P. Ginebra, Acta Biomater., 2013, 9, 8403.

20 L. Liang, P. Rulis, W.Y. Ching, Acta Biomater., 2010, 6, 3763.

21 M.I. Hasan, J.W. Bradley, J. Phys. D: Appl. Phys. 2015, 48, 435201.

22 M. Gibaldi, S. Feldman, J. Pharm. Sci., 1967, 56, 1238.

23 Joint Committee for Powder Diffraction Studies - International Center for Diffraction Data, and American Society for Testing and Materials. Powder Diffraction File. Swarthmore, PA, 1991. 